\title{
Calendar of Short Courses and Workshops Powder Diffraction March 2015
}

\author{
Gang Wang \\ Research and Development Center for Functional Crystals, Institute of Physics, Chinese Academy of Sciences, No.8 Nansanjie, Zhongguancun, \\ Haidian District, Beijing 100190, China \\ gangwang@iphy.ac.cn
}

1 March-1 April 2015

HERCULES 2015-Neutrons \& Synchrotron Radiation for Science, France and Switzerland [Info: http://hercules-school. $\mathrm{eu} /]$

\section{6-15 April 2015}

Macromolecular Crystallography School 2015: From Data Processing to Structure Refinement and Beyond, Montevideo, Uruguay [Info: http://www.iucr.org/calendar/events/types/schools/ macromolecular-crystallography-school-2015-from-data-processingto-structure-refinement-and-beyond]

\section{9-12 April 2015}

3rd School on Crystal Structure Determination from Diffraction Data: Application on Powder Samples, Sousse, Tunisia [Info: http://www.iucr.org/calendar/events/types/schools/ 3rd-school-on-crystal-structure-determination-from-diffractiondata-application-on-powder-samples]

27 April-1 May 2015

Practical X-Ray Fluorescence, Newtown Square, Pennsylvania, USA [Info: http://www.icdd.com/education/xrf.htm]

\section{4-6 May 2015}

Canadian Light Source 18th Annual Users' Meeting and Related Workshops, Saskatoon, Canada [Info: http://www.lightsource.ca/events/meeting2015/]

\section{4-10 May 2015}

EMBO Global Exchange Lecture Course-Structural and Biophysical Methods for Biological Macromolecules in Solution, Taipei, Taiwan, China [Info: http://events.embo.org/ 15-macromolecule/index.html]
18-22 May 2015

EMBO Practical Course-Small Angle Neutron and X-Ray Scattering from Proteins in Solution, Grenoble, France [Info: http://events.embo.org/15-saxs/

\section{1-5 June 2015}

Fundamentals of X-Ray Powder Diffraction, Newtown Square, Pennsylvania, USA [Info: http://www.icdd.com/education/xrd.htm]

5-14 June 2015

Engineering Crystallography: From Molecule to Crystal to Functional Form, Erice, Italy [Info: http://www.crystalerice. org/Erice2015/2015.htm]

7-20 June 2015

The Zürich School of Crystallography 2015, Zurich, Switzerland [Info: http://www.chem.uzh.ch/linden/zsc/]

8-12 June 2015

Advanced Methods in X-Ray Powder Diffraction, Newtown Square, Pennsylvania, USA [Info: http://www.icdd.com/education/xrd.htm]

\section{8-30 September 2015}

Basic Rietveld Refinement \& Indexing Workshop, Newtown Square, Pennsylvania, USA [Info: http://www.icdd.com/education/rietveld-workshop.htm]

1-2 October 2015

Advanced Rietveld Refinement \& Indexing Workshop, Newtown Square, Pennsylvania, USA [Info: http://www.icdd. com/education/rietveld-workshop.htm] 